\title{
EVALUATION OF 3D BUILDING MODEL USING TERRESTRIAL LASER SCANNING AND DRONE PHOTOGRAMMETRY
}

\author{
M. Bouziani ${ }^{1 *}$, H. Chaaba, ${ }^{1}$, M. Ettarid ${ }^{1}$ \\ ${ }^{1}$ School of Geomatic Sciences and Surveying Engineering, IAV Hassan II, BP 6202, Madinate Al Irfane, Rabat, Morocco - \\ (m.bouziani@iav.ac.ma)
}

KEY WORDS: UAV Photogrammetry, Terrestrial Laser Scanning, 3D Digital Modelling, Point Cloud, Building

\begin{abstract}
:
The objective of our study is the evaluation of the 3D modeling of buildings and the extraction of structural elements from point clouds obtained using two acquisition techniques (drone and terrestrial laser scanner), as well as the evaluation of the usefulness of their integration. The drone shooting mission was carried using the DJI Phantom 3 Professional and the Sony EXMOR 1/2.3" CMOS RGB camera. For the TLS scanning mission, 9 scanning stations were performed using the FARO Focus S350 laser scanner. To allow the fusion of the two point clouds obtained from drone imagery and TLS, an alignment step is applied. This step was performed using the Iterative Closest Point algorithm. Segmentation was performed using the adapted RANSAC algorithm on point clouds obtained from the drone mission and the TLS mission as well as on the merged point cloud in order to extract structural elements of the building such as windows, doors and stairs. Analysis of the results emphasizes the importance of TLS and drone in 3D modeling. TLS gave better results than the drone in extracting structural elements. This work confirms the importance of complementarity between these two technologies to produce detailed, complete and precise 3D models.
\end{abstract}

\section{INTRODUCTION}

Three-dimensional models of buildings represent important data sources for many applications. The efficient generation of this kind of models enables the enrichment of digital library content on buildings and infrastructure and provides managers with valuable visualization and decision support tools.

In recent years, advanced technologies have made it possible to create precise and detailed 3D models to represent buildings as built. Due to the growing need for realistic and accurate $3 \mathrm{D}$ models, Drones (UAV) and Terrestrial Laser Scanner (TLS) are emerging as essential tools for reality capture.

The images acquired by drones are used to achieve very high levels of detail in 3D models. This use allows the reconstruction of the geometry and the texture of the studied objects (Drešček et al., 2020; Achille et al., 2015; Chen et al., 2019). Drones have become widely used for data acquisition and for $3 \mathrm{D}$ reconstruction purposes. The quality of the 3D data generated strongly depends on the characteristics of the sensors used, the design of the photogrammetric network and the image orientation results.

TLS are widely used for reconstruction and $3 \mathrm{D}$ visualization. The scanner generates a dense point cloud used to produce a 3D model of the object. There are many reasons for using TLS in reality capture: detection of anomalies, site monitoring, maintenance operations, and creation of a digital BIM model (Wang et al., 2019).

The use of images acquired by drones combined with TLS acquisitions has brought many advantages to obtain complete and detailed 3D models (Farella et al., 2020). For the reconstruction of the 3D model of the building, this type of fusion can be useful, both for the detection of the construction of different angles and for the exhaustive reconstruction of the model.

Few studies have investigated the quality of the fusion of point clouds obtained by UAV images and TLS for 3D modeling. Bastonero et al. (2014) conducted a study for the evaluation of the fusion of different 3D models of the abbey church (Italy), carried out by multi-sensor techniques. When the two point clouds were integrated, it was possible to verify the potential of this union by a mesh generation with an RMS error of $5 \mathrm{~cm}$. Andaru et al. (2019) merged and integrated TLS and UAV point cloud data into a unique reference system to create complete 3D point cloud of a heritage building. In their study, they collected two architectural heritage buildings in Yogyakarta, Indonesia. Results were obtained with an RMS error of $2 \mathrm{~cm}$. The 3D models and their textures on the exterior and interior sides have been processed. The modeling was carried out on the base structure of the building façade on simple geometric primitives such as planes, straight lines, circles, spheres and cylinders.

In the following sections, we will present the proposed methodology for the evaluation of 3D building model using terrestrial laser scanning and drone photogrammetry. Then we will describe the equipment used and the study area. Then, we will give and discuss results obtained. The article ends with the conclusion.

\footnotetext{
* Corresponding author
} 


\section{METHODOLOGY}

The main objective of this project is to evaluate the 3D modeling of a building and the extraction of structural elements (windows, doors, stairs) from point clouds obtained from drone RGB images and TLS. The case study is the great Amphitheater of Agronomy and Veterinary Institute Hassan II (Rabat, Morocco). This building was built in 2016. It has an approximate area of $3900 \mathrm{~m} 2$ with a capacity of around 400 places. It is characterized by its oval outer shape and its annexes of different geometric shapes. The amphitheater has 8 doors and 13 windows distributed on its exterior. The following figure shows the study area.

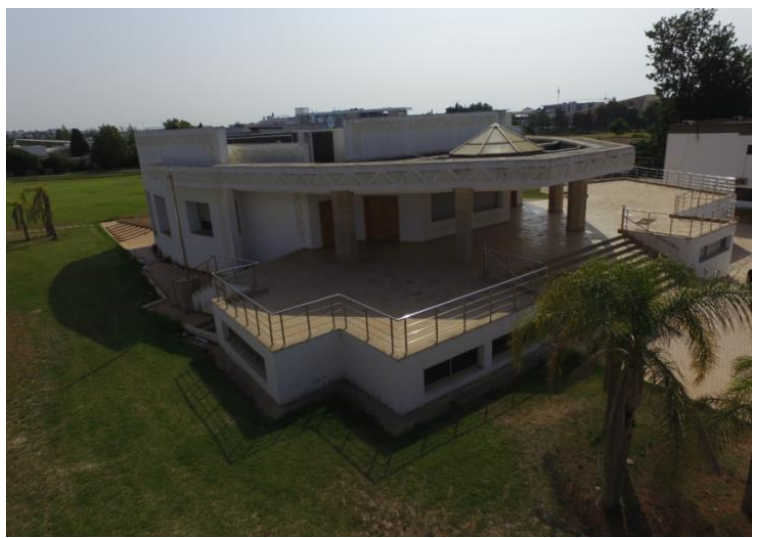

Figure 1. The great Amphitheater of Agronomy and Veterinary Institute Hassan II (Rabat, Morocco).

The drone shooting mission was carried out on May 26, 2018. The drone used is the Phantom 3 Professional from the DJI brand. The shooting was carried out with the Sony EXMOR 1/2.3" CMOS RGB camera. A circular flight mission planned with the Pix4D-Capture application was carried out with a flight height ranging from $10 \mathrm{~m}$ to $60 \mathrm{~m}$ and images acquired every 6 degrees. The selected frontal and side overlap is 70\%. 263 images have been acquired. The processing includes the following steps: camera alignment, generation of dense point clouds, creation of meshes, generation of textures and finally creation of 3D models. This processing was done on Agisoft's Metashape.

For the building's TLS scanning mission, checkered targets were used to ensure good consolidation of the scans. 9 scanning stations were performed using the FARO Focus S350 laser scanner. The average scanning time per scan is 4 minutes with an overall point cloud of 11 million points and a point distance of $12 \mathrm{~mm} / 10 \mathrm{~m}$. Points acquired by TLS goes through the following steps: cleaning, consolidation, creation of the mesh, texture of the mesh model and finally creation of the 3D model. FaroSCENE and 3DReshaper software were used.
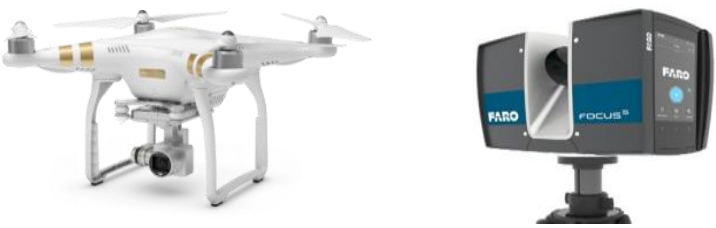

Figure 2. Equipment used: Phantom 3 Professional and Faro Focus S350
To allow the fusion of the two point clouds, an alignment step is necessary. This step is performed using the ICP (Iterative Closest Point) algorithm. The transformation parameters of the two point clouds are calculated through the relationship between the corresponding matching points to satisfy the convergence precision. Then, the translation and the rotation parameters between the two point clouds are obtained to complete the fusion process.

The initial values of the iteration are determined by performing many tests. This step is critical. If the initial values are not appropriate, the ICP algorithm may lead to a local optimum and the iteration cannot converge to the correct fusion result (He et al., 2017). The algorithm has four steps: (1) Point selection to find the nearest points in the two point clouds. A down sampling operation is performed to accelerate this step. (2) Retrieving corresponding points to find the nearest point from another point set as the corresponding point of the current point. An appropriate rapid search algorithm is used based on kd-tree data structure. (3) Point pair exclusion by defining error point to improve the fusion accuracy. (4) Defining error metrics function to minimize errors and improve the accuracy of the fusion result. This registration was performed with a final RMS error of $6 \mathrm{~cm}$ and an overlap of $100 \%$.

\section{RESULTS AND DISCUSSION}

The following figure shows the three point clouds obtained.

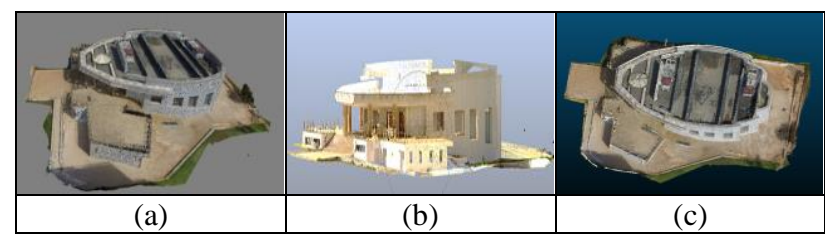

Figure 3. Dense point clouds obtained: (a) by drone imagery, (b) by TLS, (c) Fusion of the two previous point clouds

The model resulting from the drone point cloud lacks information on the entrance to the amphitheater because of the distance and the angle of view of the aerial images. On the other hand, it gives a complete model of the roof of the building. The point cloud of the TLS gives the possibility to model the façades and the entrance with a lot of detail and a better precision. The merged model offers complete information on these two parts of the amphitheater.

We realized a cloud-cloud comparison on the totality of the two point clouds obtained by drone and by TLS. The deviation mapping generated shows an average difference of $0.15 \mathrm{~m}$ and a standard deviation of $0.11 \mathrm{~m}$.

To estimate the accuracy of the 3D model obtained by TLS, we compared the distances between the point cloud obtained by TLS and the mesh model using the "Cloud to Mesh Distance" function of the CloudCompare software. We obtained an average deviation of $2 \mathrm{~mm}$ and a standard deviation of $1 \mathrm{~cm}$.

Using the same function mentioned above, we made an analysis this time for the point cloud obtained by drone imagery in comparison with the reference mesh model. The comparison results give an average distance of $1 \mathrm{~cm}$ and a standard deviation equal to $9 \mathrm{~cm}$.

Then, we proceeded to evaluate the merged model. After comparing the point cloud merged from the two sources with the mesh model, we obtained an average distance of $3 \mathrm{~mm}$ and a standard deviation equal to $2 \mathrm{~cm}$. We can therefore say that the 
integration of the two point clouds improves the accuracy of the three-dimensional modeling of the amphitheater.

We then performed segmentation tests on the point clouds obtained from the drone mission and the TLS mission as well as on the merged point cloud using the adapted Random Sample Consensus (RANSAC) algorithm. We did the segmentation by varying the minimum number of points on different combinations of geometric shapes such as planes, spheres, cylinders and cones.

The adapted RANSAC is an efficient optimization method used to recognize geometric shapes from a set of points, despite the presence of noise and outliers. The method is iterative; the recognition begins with the random sampling of a minimal number of points to estimate the parameters of the shape. The set of points at a certain distance from the model are then appointed inliers while the rest of the points are outliers (Ait Elkadi et al., 2013).

This segmentation gives the possibility of extracting several structural elements of the building such as windows, doors and stairs. The following figure shows the result of the segmentation.

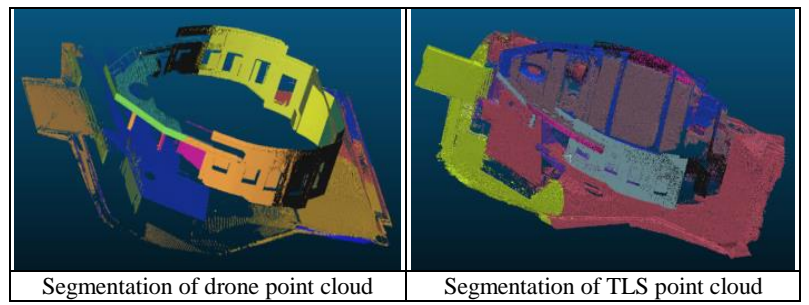

Figure 4. Segmentation Results

For the extraction of structural elements on façades such as windows, stairs and doors, the use of the point cloud resulting from the TLS gives more interesting results compared to the one resulting from the drone images.

The point cloud obtained from the drone images does not give the requested visual quality and this is explained by its low precision which complicates the choice of parameters and the process of extraction by the RANSAC algorithm.

Merging the two point clouds improves extraction. The quality of the integration of the two point clouds depends on the complexity of the geometry of the structure to be extracted. For example for the doors and the stairs of the entrance which have a simple planar geometry we obtain a visual improvement in comparison with the two source point clouds, but we find a difficulty in identifying the side windows since they have a complex curved geometry.

The following figures show examples of the extraction results. From these figures, we notice that the point cloud obtained from TLS gives a better extraction of the façade elements. From the segmentation of this point cloud, we can clearly identify the different structural elements to be extracted. However we see that the point cloud obtained from drone imagery does not give the visual quality requested on the façades and this comes down to its low precision which complicates the choice of parameters and the extraction process by the RANSAC algorithm.

On the other hand, the quality of the integration of the two point clouds depends on the complexity of the geometry of the structure to be extracted. For example for the entrance doors and stairs which have a simple planar geometry we obtain an improvement compared to the two source point clouds, but we find difficulty in identifying the side windows as they have a complex geometry.
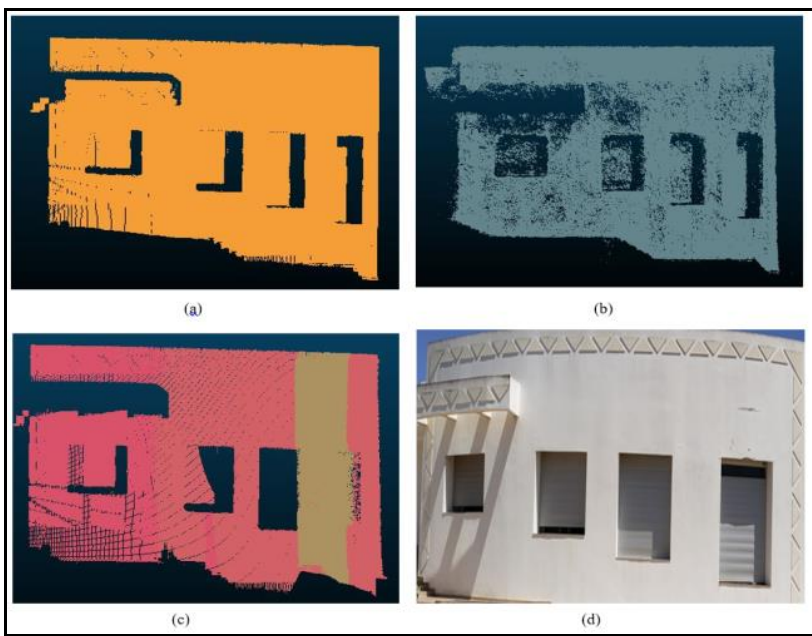

Figure 5. Extraction of windows from different point clouds: (a) from TLS, (b) from Drone, (c) from merged point cloud, (d) origin image
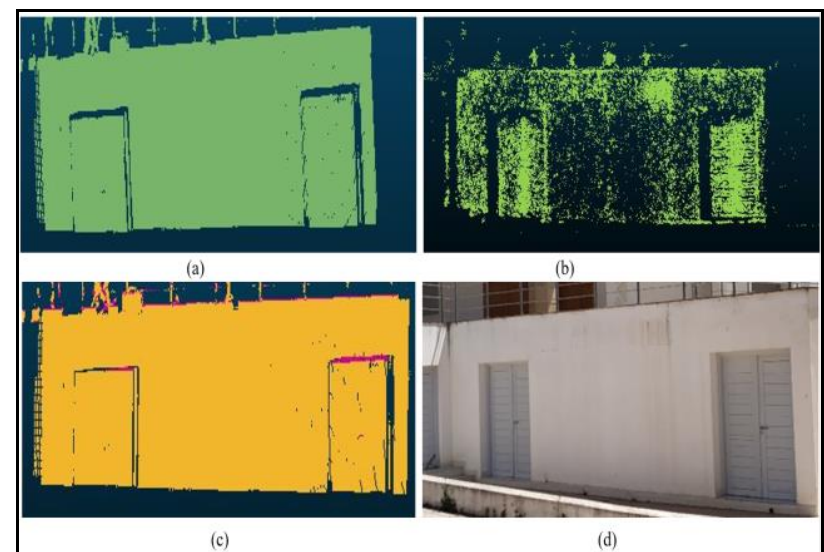

Figure 6. Extraction of doors from different point clouds: (a) from TLS, (b) from Drone, (c) from merged point cloud, (d) origin image

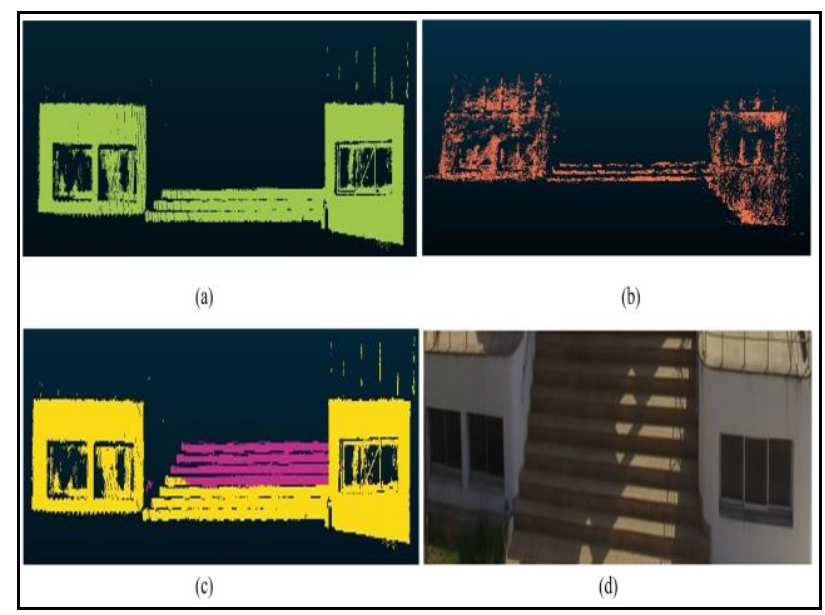

Figure 7. Extraction of the Stairs at the entrance to the building: (a) from TLS, (b) from Drone, (c) from merged point cloud, (d) origin image 
Further experiments are underway to improve the integration of the two technologies for other types of buildings for the evaluation of the digitization of buildings and to generate digital BIM models.

\section{CONCLUSION}

Our work consisted in the evaluation of the 3D modeling and the extraction of structural elements from point clouds from two technologies, namely: drone photogrammetry and terrestrial laser scanning, as well as the evaluation of the contribution of their integration.

At the end of this work, we found that each technique has strengths and limitations. Regarding the drone technique, its advantage is having a very dense point cloud, which gives a model that is faithful to reality. Thus, this method offers the possibility of modeling the roof and the facades thanks to the different angles of view. However, the generation of the point cloud is not done directly but based on the image matching algorithm which generates a dense point cloud but with errors that accumulate during the processing process. This imprecision is due to the georeferencing quality of the point cloud. Also, the time required to process drone images and the high configurations required for the workstation represent a very significant limit.

As for the terrestrial laser scanner, the direct acquisition of the point cloud in the field represents a strong point of this technique. It allows having a sufficient density of points to model the facades of the building and the extraction of the elements of its structure. However, to have full information on the facades of the building and its elements, we need to do several scans.

The integration of the two sets of point clouds improves the completeness of the coverage, which allows the modeling of the complex objects of this building. If drone oblique images are not available, we can use the TLS to capture the facades and the drone nadir images for the roof and integrate them to have full coverage on the building.

For the extraction of structural elements such as windows, stairs and doors, and from our work in which we have exploited the geometric segmentation by the RANSAC algorithm, we can affirm that the use of the terrestrial laser scanner gives more interesting results compared to the point cloud resulting from drone images. However, merging the two point clouds improves the extraction results.

\section{REFERENCES}

Achille, C., Adami, A., Chiarini, S., Cremonesi, S., Fassi, F., Fregonese, L. and Taffurelli, L., 2015. UAV-based photogrammetry and integrated technologies for architectural applications-Methodological strategies for the after-quake survey of vertical structures in Mantua (Italy). Sensors, 15(7), pp.15520-15539.

Aitelkadi, K., Tahiri, D., Simonetto, E., Sebari, I., Polidori, L., 2013. Segmentation of heritage building by means of geometric and radiometric components from terrestrial laser scanning. ISPRS Annals of the Photogrammetry, Remote Sensing and Spatial Information Sciences, Volume II-5/W1, 2013, XXIV International CIPA Symposium, Strasbourg, France, 2013.
Andaru, R., Cahyono, B. K., Riyadi, G., Istarno, Djurdjani, Ramadhan, G. R., Tuntas, S., 2019. "The combination of terrestrial Lidar and UAV photogrammetry for interactive architectural heritage visualization using unity 3D game engine", Int. Arch. Photogramm. Remote Sens. Spatial Inf. Sci, Volume XLII-2/W17, 2-3 December 2019, Strasbourg, France, pp 39-44.

Bastonero, P., Donadio, E., Chiabrando, F., Spanò, A., 2014. Fusion of 3D models derived from TLS and image-based techniques for $\mathrm{CH}$ enhanced documentation, ISPRS Annals of Photogramm. Remote Sens. Spatial Inf. Sci, Volume II-5, 2014 ISPRS Technical Commission V Symposium, 23 - 25 June 2014, Riva del Garda, Italy pp 73-80.

Chen, S., Laefer, D.F., Mangina, E., Zolanvari, S.I. and Byrne, J., 2019. UAV bridge inspection through evaluated 3D reconstructions. Journal of Bridge Engineering, 24(4), p.05019001.

Drešček, U., Fras M. K., Tekavec, J., Lisec, A., 2020. Spatial ETL for 3D Building Modelling Based on Unmanned Aerial Vehicle Data in Semi-Urban Areas. Remote Sensing, 12(12), 1972.

Farella, E. M., Torresani, A., Remondino, F., 2020. Refining the Joint 3D Processing of Terrestrial and UAV Images Using Quality Measures. Remote Sensing, 12(18), 2873.

He, Y., Liang, B., Yang, J., Li, S., He, J., 2017. An Iterative Closest Points Algorithm for Registration of 3D Laser Scanner Point Clouds with Geometric Features. Sensors, 17(8), 1862.

Wang, Q., Kim, M.K., 2019. Applications of 3D point cloud data in the construction industry: A fifteen-year review from 2004 to 2018. Advanced Engineering Informatics, 39, 306-319. 